University of Nebraska - Lincoln

DigitalCommons@University of Nebraska - Lincoln

Roger Kirby Publications

Research Papers in Physics and Astronomy

February 1971

Far-Infrared Properties of Lattice Resonant Modes. V. SecondOrder Stark Effect

B.P. Clayman

University of Nebraska-Lincoln

Roger D. Kirby

University of Nebraska-Lincoln, rkirby1@unl.edu

A. J. Sievers

University of Nebraska-Lincoln

Follow this and additional works at: https://digitalcommons.unl.edu/physics_kirby

Part of the Physics Commons

Clayman, B.P.; Kirby, Roger D.; and Sievers, A. J., "Far-Infrared Properties of Lattice Resonant Modes. V. Second-Order Stark Effect" (1971). Roger Kirby Publications. 32.

https://digitalcommons.unl.edu/physics_kirby/32

This Article is brought to you for free and open access by the Research Papers in Physics and Astronomy at DigitalCommons@University of Nebraska - Lincoln. It has been accepted for inclusion in Roger Kirby Publications by an authorized administrator of DigitalCommons@University of Nebraska - Lincoln. 
Phys. Rev. Letters 23,226 (1969).

${ }^{11} \mathrm{D}$. Eisenberg and W. Kauzmann, Structure and Properties of Water (Oxford U. P., Oxford, England, 1969).

${ }^{12}$ T. S. Moss, Optical Properties of Semiconductors (Butterworths, London, 1961).

${ }^{13}$ S. Bardasis and D. Hone, Phys. Rev. 153, 849 (1967).

${ }^{14} \mathrm{E}$. W. Aslaksen, Phys. Rev。 Letters 24, 767 (1970).

${ }^{15} \mathrm{C}$. Y. Fong and M. L. Cohen, Phys. Rev. $\underline{185}, 1168$ (1968).

${ }^{16}$ C. Y. Fong, W. Saslow, and M. L. Cohen, Phys。
Rev. 168, 992 (1968).

${ }^{17}$ D. Brust, Phys. Rev. 134, A1337 (1964).

${ }^{18} \mathrm{H}$. R. Philipp and H. Ehrenreich, Phys. Rev. $\underline{129}$, 1550 (1963).

${ }^{19}$ S. H. Wemple and M. DiDomenico, Jr., Applied

Solid State Science, edited by R. Wolfe (Academic, New

York, to be published), Vol. III.

${ }^{20}$ S. H. Wemple and M. DiDomenico, Jr., Phys. Rev. B 1,193 (1970).

${ }^{21}$ R. W. Shaw, Jr., Phys. Rev. Letters 25 , 818 (1970).

\title{
Far-Infrared Properties of Lattice Resonant Modes. V. Second-Order Stark Effect*
}

\author{
B. P. Clayman \\ Laboratory of Atomic and Solid State Physics, Cornell University, Ithaca, New York 14850 \\ and \\ Department of Physics, Simon Fraser University, Burnaby 2, British Columbia, Canada ${ }^{\dagger}$ \\ and \\ R. D. Kirby ${ }^{\ddagger}$ and A. J. Sievers ${ }^{\S}$ \\ Laboratory of Atomic and Solid State Physics, Cornell University, Ithaca, New York 14850 \\ (Received 8 September 1970)
}

Small electric-field-induced frequency shifts have been observed for resonant modes associated with three defect systems. For NaI: $\mathrm{Cl}^{-}$, the shifts have been used to measure the quartic anharmonic terms of the interionic potential of the impurity ion. For $\mathrm{KBr}: \mathrm{Li}^{+}$, the quartic anharmonic terms are found to be very small, and an harmonic potential which includes a central barrier with the barrier height less than the zero-point energy of the oscillator is required to explain the experimental results. For $\mathrm{NaCl}: \mathrm{Cu}^{+}$, only an "on-center" resonant-mode configuration is consistent with the experimental results.

\section{INTRODUCTION}

The response of lattice resonant modes in alkalihalide crystals to an external dc electric field is a sensitive probe of the local impurity environment. For a harmonic-oscillator resonant mode associated with an "on-center" defect, an applied electric field shifts all energy levels by the same amount, and no change in the far-infrared absorption frequency is to be expected. For paraelectric impurities, whose far-infrared properties are strongly modified by the tunneling motion of the "off-center" impurity ion, giant electric field effects have been observed. ${ }^{1}$ We have measured small electricfield-induced frequency shifts associated with three "on-center" defect systems: $\mathrm{NaI}: \mathrm{NaCl}, \mathrm{KBr}: \mathrm{LiBr}$, and $\mathrm{NaCl}: \mathrm{CuCl}$. Because these experiments complement previous far-infrared measurements incorporating other perturbations, ${ }^{2,3}$ some definite features of the anharmonic potentials which bind these impurities can now be resolved.

Of the three lattice-defect systems, the largest electric-field-induced frequency shifts have been observed in NaI: $\mathrm{Cl}^{-}$. The resonant-mode frequency shifts have been used to determine the cubic symmetry of the defect site and to measure the quartic anharmonic terms of the interionic potential of the impurity ion. A preliminary description of these findings has been given earlier. ${ }^{4}$

The much smaller electric-field-induced shifts ${ }^{5}$ observed for $\mathrm{KBr}: \mathrm{Li}^{+}$are significant in that they eliminate the possibility that quartic anharmonic terms play an important role in the local potential of this defect. Both the small electric field shift and the large isotope shift previously observed ${ }^{2}$ are explained with an harmonic potential containing a central barrier. The barrier is small compared to the zero-point energy of the resonant mode, hence the impurity still appears to occupy the normal equilibrium lattice site.

The null electric field effects observed for $\mathrm{NaCl}: \mathrm{Cu}^{+}$are consistent with an "on-center" resonant-mode configuration. For this case, the exact shape of the potential has not been determined. 

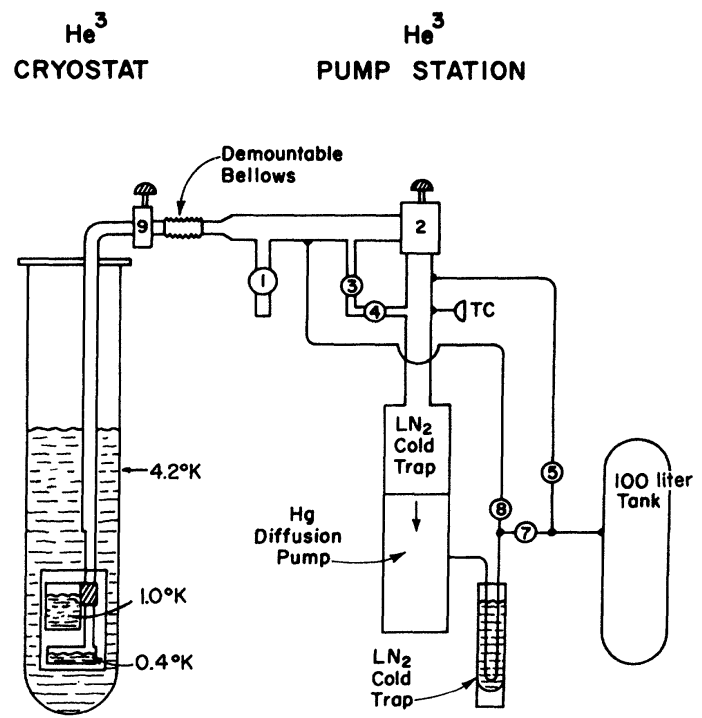

FIG. 1. $\mathrm{He}^{3}$ handling system. Bellows valves 1-9 control the gas flow. TC denotes a thermocouple gauge head. An Edwards No. 2M4A diffusion pump is the key component.

In Sec. II, we describe some far-infrared techniques including a $\mathrm{He}^{3}$-cooled-detector cryostat. The experimental results are given in Sec. III. In Sec. IV, a model of a resonant mode in which the impurity ion moves against the rest of the lattice is introduced. The experimental results are compared with the frequencies calculated from an expansion of the potential energy for small displacements of the impurity ion consistent with the $O_{h}$ site symmetry. We conclude Sec. III with a onedimensional calculation of an harmonic oscillator containing a Gaussian-shaped barrier with a height which is smaller than the zero-point energy of the oscillator. Good agreement is obtained between this model calculation and the experimental results of $\mathrm{KBr}: \mathrm{Li}^{+}$.

\section{EXPERIMENTAL APPARATUS AND TECHNIQUES}

\section{A. Detector-Sample Cryostat}

The majority of the far-infrared transmission measurements were made using a Strong-type lamellar interferometer. ${ }^{6}$ A microwave harmonic generator operating at $5.5 \mathrm{~cm}^{-1}$ provided corroborative data on the $\mathrm{NaI}: \mathrm{NaCl}$ system. ${ }^{7} \mathrm{~A} \mathrm{He}^{3}$-cooled bolometer, somewhat different in design and operation from that described by Drew and Sievers, ${ }^{8}$ served as the radiation detector for all the measurements.

The entire $\mathrm{He}^{3}$ system is schematically represented in Fig. 1. The $\mathrm{He}^{3}$ pump line is attached to the cryostat by means of a bellows connection to a separate pump station, which contains a mercury diffusion pump and associated vacuum plumbing. The separate pump-station design permits the use of the same $\mathrm{He}^{3}$ pump and components with other $\mathrm{He}^{3}$ evaporators. During $\mathrm{He}^{3}$ condensation, valves 5 and 8 are opened. The condensation rate is limited by the $10-\mathrm{cm}^{2}$ surface of oxygen-free highconductivity (OFHC) copper cooled to $1^{\circ} \mathrm{K}$ by the $\mathrm{He}^{4}$ tank. $4 \mathrm{~cm}^{3}$ of liquid $\mathrm{He}^{3}$ condenses in $15 \mathrm{~min}$. Once it is condensed all valves are closed, then valves 3 and 7 are opened. Throttle valve 4 is opened gradually as the $\mathrm{He}^{3}$ section is cooled. Finally, valve 2 is opened to obtain full pumping speed.

It has been found that continuous operation at $0.35^{\circ} \mathrm{K}$ from 8 to $20 \mathrm{~h}$ is obtained for this system, depending upon the heat input through quartz vacuum window shown in Fig. 2. This heat input is determined by the filtering used in the cryostat and by the temperature of the samples. For those runs in which the high-frequency limit was $100 \mathrm{~cm}^{-1}$ and the samples were at $4.2{ }^{\circ} \mathrm{K}$, the period of operation was much closer to the upper limit than the lower one. To ensure a $100-\mathrm{cm}^{-1}$ upper limit on the background radiation, a composite filter consisting of thin beveled slabs of $\mathrm{LiF}, \mathrm{NaCl}$, and $\mathrm{KI}$ was inserted in the $4.2{ }^{\circ} \mathrm{K}$ section of the light pipe.

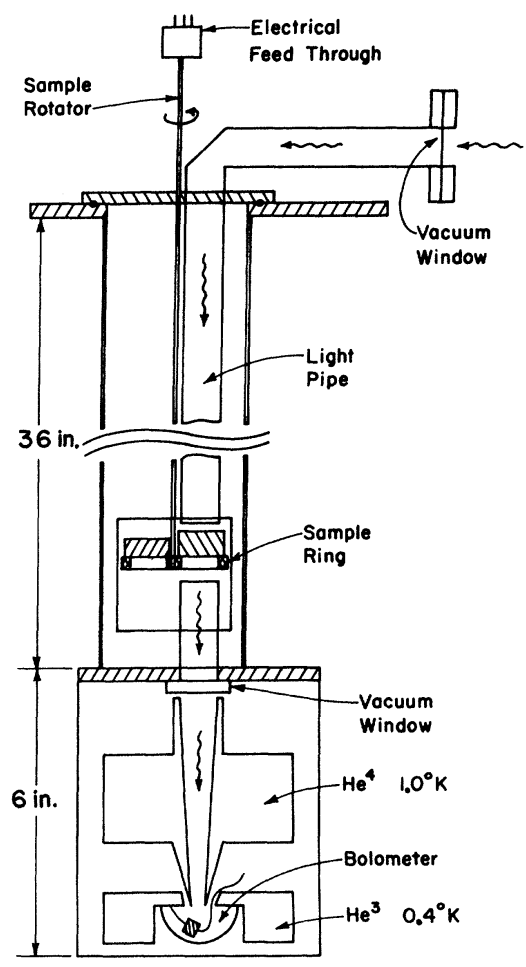

FIG. 2. Sample-detector cryostat. Over-all view of the cryostat, emphasizing the details of the sample section. Samples are shown in place on the sample ring. The cryostat is immersed in liquid helium. 


\section{Sample Section}

The section of the cryostat which contains the samples is directly above the detector section, separated from it by the quartz vacuum window as shown schematically in Fig. 2. It consists of a $90 \times 4$. 75-cm-diam thin-wall stainless-steel tubing extending from the top of the cryostat down into the outer $\mathrm{He}^{4}$ bath to the detector section. It may be partially or completely evacuated, or it may be opened directly to the outer bath and filled with liquid $\mathrm{He}^{4}$ to the depth of the outer bath. Thus, the sample-holding insert may be removed from the cryostat without disturbing the detector system. This configuration permits consecutive use of the cryostat for several different experiments without the necessity of warming it above liquid-helium temperatures.

Shown in Fig. 2 is the insert of measuring the bulk absorption in alkali-halide samples. Radiation enters the cryostat through a polyethylene vacuum window. It is transmitted down to the sample section through a 1.4-cm-diam stainless-steel light pipe, gold plated on the inside. The samples typically 2-15 $\mathrm{mm}$ thick, are fastened over $1.25-\mathrm{cm}$-diam holes in a copper sample ring by means of phosphor-bronze clips. These four-position sample rings can be rotated from the top of the cryostat to position the desired sample in the beam. This per-

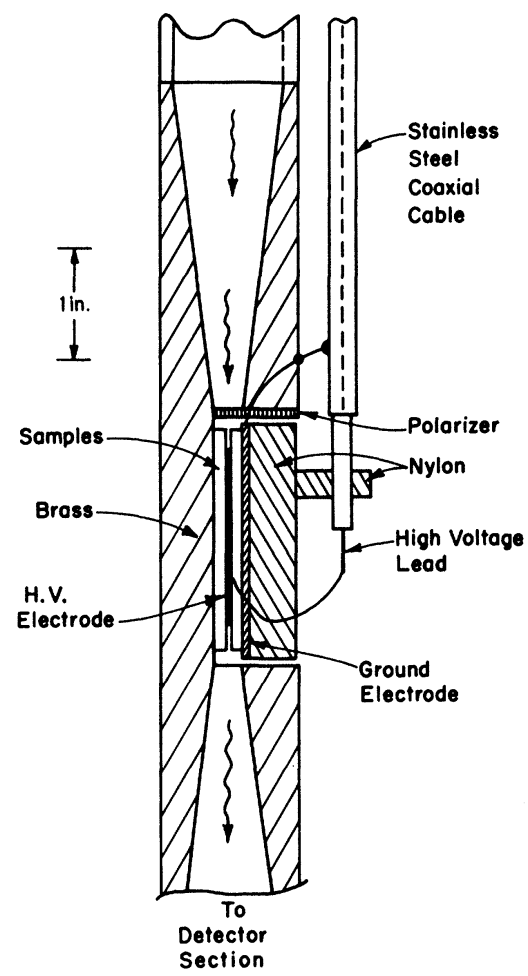

FIG. 3. Cross-section view of the electric field insert.

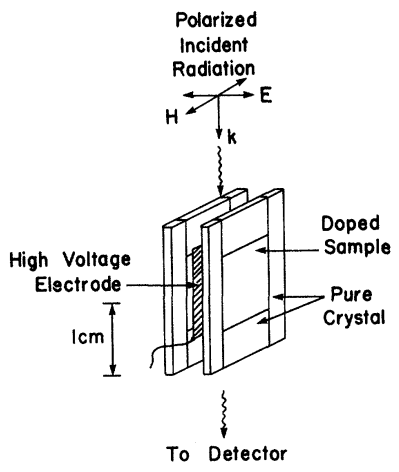

FIG. 4. Exploded view of the composite electric field sample.

mits the transmission spectra of pure and doped samples to be compared directly and the impurity induced absorption determined.

For temperature-dependence studies, the sample section is sealed and coupled to the outer bath via helium exchange gas. A heater is wound around the sample ring and a carbon resistor is placed in thermal contact with the samples. Heater and thermometer leads are carried through the rotator rod to an electric feed-through at the top of the cryostat.

The electric field sample holder is shown in Fig. 3. The light beam is reduced from a $1.25 \mathrm{~cm}$ diam to a $0.32 \times 1.25-\mathrm{cm}$ window in a tapered section. A polarizer consisting of a fine-wire grid deposited on Mylar film is mounted in the light path at the end of the tapered section preceding the sample. The complete sample is pressed against the body of the sample holder by a brass ground electrode and a nylon retainer. Below the sample, the 0.32 $\times 1.25-\mathrm{cm}$ window tapers back to $1.25 \mathrm{~cm}$ diam and this light pipe leads directly to the detector section.

In order to minimize the effects of field inhomogeneity, a special sample configuration was devised. It is shown in Fig. 4. The doped sample is framed by four pieces of pure crystal in which no impurityinduced absorption takes place. The fringing fields of this parallel-plate arrangement occur mostly in the pure crystal so that the field on the doped sample is uniform.

\section{Detector Section}

Probably the most significant improvement over the previous refrigerator design ${ }^{8}$ is the additional internal pumped $\mathrm{He}^{4}$ bath which condenses the $\mathrm{He}^{3}$ gas initially and thermally isolates the $\mathrm{He}^{3}$ bath from the outer $4.2{ }^{\circ} \mathrm{K}$ bath. This isolation greatly increases the capacity for continuous operation and makes the bolometer insensitive to changes in the temperature of the outer bath.

The section of the cryostat housing the bolometer is shown schematically in Fig. 5. A 1.3-cm-diam quartz vacuum window admits far-infrared radiation from the sample section. A polished-copper 


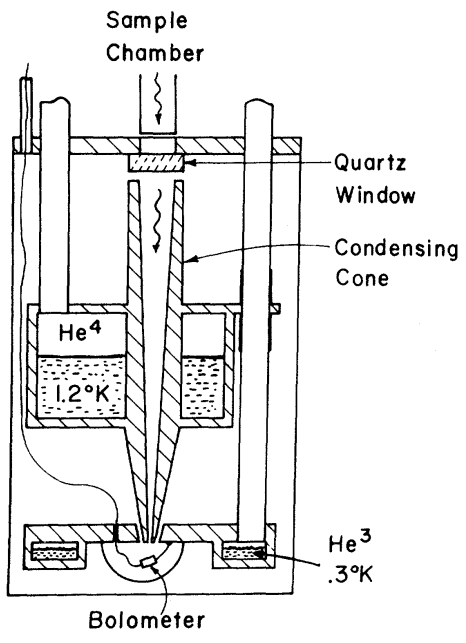

FIG. 5. Cross-section view of the cryostat housing the bolometer. Bolometer lead is carried to the top of the cryostat through the pump-out tube.

light cone condenses the beam to the 4-mm-diam entrance of the hemispherical 2.5-cm-diam cavity which houses the bolometer. The liquid $\mathrm{He}^{3}$ is held in a ring-shaped tank, shown in cross section in Fig. 5. Liquid $\mathrm{He}^{4}$ is held in the upper tank, whose volume is $\sim 70 \mathrm{~cm}^{3}$. It is filled via a needle valve which opens the pump line to the outer bath; the valve is operated from outside the cryostat. Both tanks are pumped on through $1.25-\mathrm{cm}$ stainlesssteel tubes. A section of the $\mathrm{He}^{3}$ pump line is in thermal contact with the $\mathrm{He}^{4}$ tank; condensation of the $\mathrm{He}^{3}$ gas occurs in this section, once the upper tank is pumped to $1.0^{\circ} \mathrm{K}$.

\section{B. Measurement Techniques}

All electric field measurements were made with the sample holder immersed in liquid helium at $4.2{ }^{\circ} \mathrm{K}$. The high-voltage source was a $0-30-\mathrm{kV}$ Sorenson 1030-20 power supply. In series with the samples were a $30-\mathrm{M} \Omega$ resistor and a 0-16 microammeter. In this way the current (if any) passing through the samples could be measured. It was found that, when using this $\mathrm{NaI}$ : $\mathrm{NaCl}$ sample, applied voltages of $8 \mathrm{kV}$ could be applied with no leakage, and $12 \mathrm{kV}$ could be applied with a leakage current about $3 \mu \mathrm{A}$. In this case, more than $99 \%$ of the applied voltage appears across the samples. Higher voltages caused intermittent breakdown, although $16 \mathrm{kV}$ could be applied for periods of several minutes before breakdown occurred.

The far-infrared transmission spectra of the samples were measured for a number of different values of electric field, applied in the [100] and [110] crystal directions. Incident radiation $\left(E_{\mathrm{IR}}\right)$ was polarized either parallel or perpendicular to the applied field for $E_{d c} \|[100]$. For $E_{d c} \|[110]$, in- cident radiation was polarized in the [110], [1 $\overline{1} 0]$, or [001] direction.

Because of the impossibility of making a pure (reference) sample with the same geometrical properties as the doped one, it was necessary to determine the shape and frequency of the field-shifted absorption lines by an indirect method. The transmission spectrum with field $E$ applied $I(E, \nu)$ was first divided by the unshifted zero-field spectrum $I(O, \nu)$. The electric-field-induced absorption coefficient $\alpha(E, \nu)$ was then formed:

$$
I(E, \nu) / I(O, \nu)=e^{-\alpha(E, \nu) t},
$$

where $t$ is the sample thickness. We add the absorption coefficient of the unperturbed line $\alpha(O, \nu)$ to $\alpha(E, \nu)$ to obtain the desired result.

Except for the method of detection, the measurements with the microwave harmonic generator are conducted in a manner similar to the paraelectric resonance experiments used to study dipolar impurities in alkali halides. ${ }^{9,10}$ The $1.8-\mathrm{cm}^{-1}$ klystron output is incident on a point-contact diode, which serves as the nonlinear element in generating the microwave harmonics. The particular harmonic desired (for NaI: $\mathrm{C1}^{-}$, the third harmonic at $\sim 5.4$ $\mathrm{cm}^{-1}$ is used) is isolated using a far-infrared grating spectrometer. The transmission of this radiation through the sample is then measured with the bolometer. By sweeping the strength of the external electric field, the resonant-mode absorption frequency could be shifted through the third harmonic frequency, allowing the applied field corresponding to the maximum absorption to be determined. ${ }^{7}$

\section{EXPERIMENTAL RESULTS}

\section{A. $\mathrm{Nal}: \mathrm{NaCl}$}

$\mathrm{NaI}: \mathrm{Cl}^{-}$has a low-lying resonant mode at 5.4 $\mathrm{cm}^{-1}$, ${ }^{11}$ with a linewidth (full width at half-maximum absorption) of $0.17 \mathrm{~cm}^{-1}$. Some broadening occurs for the higher-impurity concentrations. The absorption frequency also has a weak dependence on the $\mathrm{Cl}^{-}$concentration, which must be taken into account in the analysis of the electric field results.

Typical results of the data-reduction procedure described earlier are shown in Fig. 6 for $\mathrm{NaI}: \mathrm{NaCl}^{37}$ $(0.12 \%)$. $0-$ and $53-\mathrm{kV} / \mathrm{cm}$ applied fields are compared for $E_{\mathrm{IR}}\left\|E_{\mathrm{dc}}\right\|[100]$. The absorption line shifts upwards in frequency and broadens considerably upon application of the field. The absorption strength remains approximately constant. The results of the NaI: $\mathrm{NaCl}$ runs are summarized in Fig. 7 for $E_{\mathrm{dc}} \|[100]$ and in Fig. 8 for $E_{\mathrm{dc}} \|[110]$. The zero-field absorption frequency in Fig. 8 is 5.54 $\mathrm{cm}^{-1}$; the crystal contained $0.15 \% \mathrm{NaCl}$.

The linear fit shown in Fig. 7 represents a dependence of the frequency shift on $V^{n}$, where $V$ is the applied voltage and $n=2.0 \pm 0.1$. This quadratic 


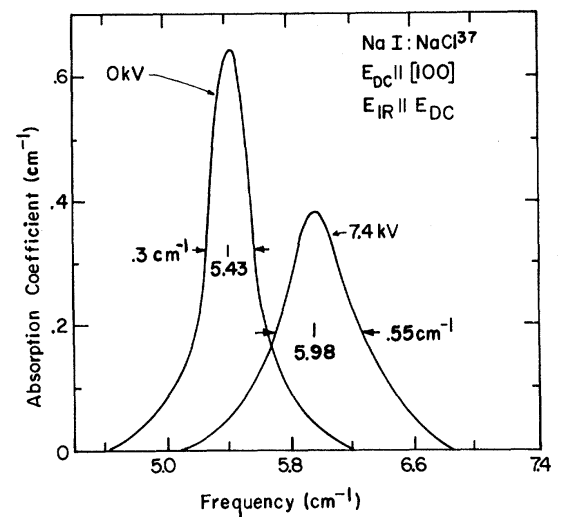

FIG. 6. Electric-field-induced shift of the resonant mode. Sample is at $4.2^{\circ} \mathrm{K}$ and the instrumental resolution is $0.18 \mathrm{~cm}^{-1}$.

dependence is also seen in Fig. 8. The slopes of the absorption frequency vs $E_{\text {external }}{ }^{2}$ curves are given in Table I for both field directions and all polarizations.

The linewidth in $\mathrm{NaI}: \mathrm{NaCl}$ increases quadratically with field from about $0.3 \mathrm{~cm}^{-1}$ at zero field to about $1.0 \mathrm{~cm}^{-1}$ at $86-\mathrm{kV} / \mathrm{cm}$ applied field for $E_{\mathrm{IR}}\left\|E_{\mathrm{dc}}\right\|$ [100]. The broadening is also observed for the data presented in Fig. 8. However, no broadening is observed for $E_{\mathrm{IR}} \|[001]$ for which the shift is nearly zero. The integrated absorption strength is found to be constant with field, within the estimated uncertainty of $\pm 20 \%$. This is illustrated in Fig. 9 for $E_{\mathrm{IR}}\left\|E_{\mathrm{dc}}\right\|[100]$.

In the microwave absorption experiments on $\mathrm{NaI}: \mathrm{NaCl}$, the applied field corresponding to maximum absorption was determined. As the dc voltage was increased from 0.0 to $10.0 \mathrm{kV}$ in $100-\mathrm{V}$ steps, the transmitted intensity varied smoothly. No fine structure was seen above the $10 \%$ noise level. Noise and drifts in the harmonic generator output limited the accuracy of the determinations of the peak absorption to $\pm 0.3 \mathrm{kV}$. The slope obtained from these measurements for $E_{\mathrm{IR}}\left\|E_{\mathrm{dc}}\right\|[100]$ is

TABLE I. Experimentally determined quadratic frequency shifts.

\begin{tabular}{|c|c|c|c|}
\hline \multirow[t]{2}{*}{ Sample } & \multicolumn{2}{|c|}{ Polarization } & \multirow{2}{*}{$\begin{array}{c}\hbar \Delta \Omega / E^{2} \\
\left(10^{-4}\right)\left[\mathrm{cm}^{-1} /(\mathrm{kV} / \mathrm{cm})^{2}\right]\end{array}$} \\
\hline & $E_{\mathrm{dc}}$ & $E_{\mathrm{IR}}$ & \\
\hline $\mathrm{NaI}: \mathrm{NaCl}^{37}$ & $\begin{array}{l}{[100]} \\
{[100]}\end{array}$ & $\begin{array}{l}{[100]} \\
{[001]}\end{array}$ & $\begin{array}{r}2.04 \pm 0.04 \\
-0.04 \pm 0.02\end{array}$ \\
\hline $\mathrm{NaI}: \mathrm{NaCl}$ & $\begin{array}{l}{[110]} \\
{[110]} \\
{[110]}\end{array}$ & $\begin{array}{l}{[110]} \\
{[1 \overline{1} 0]} \\
{[001]}\end{array}$ & $\begin{array}{c}0.705 \pm 0.04 \\
1.23 \pm 0.04 \\
-0.02 \pm 0.01\end{array}$ \\
\hline $\mathrm{KBr}: \mathrm{Li}^{7} \mathrm{Br}$ & $\begin{array}{l}{[100]} \\
{[100]}\end{array}$ & $\begin{array}{l}{[100]} \\
{[001]}\end{array}$ & $\begin{array}{r}0.16 \pm 0.06 \\
-0.11 \pm 0.06\end{array}$ \\
\hline
\end{tabular}

$(2.4 \pm 0.4) \times 10^{-4} \mathrm{~cm}^{-1} /(\mathrm{kV} / \mathrm{cm})^{2}$. This agrees well with the value of $(2.04 \pm 0.04) \times 10^{-4} \mathrm{~cm}^{-1} /(\mathrm{kV} / \mathrm{cm})^{2}$ found from the interferometer measurements.

\section{B. $\mathrm{KBr}: \mathrm{Li}^{7} \mathrm{Br}$}

The ${ }^{7} \mathrm{Li}^{+}$resonant mode in $\mathrm{KBr}$ is at $16.1 \mathrm{~cm}^{-1} .^{2}$ In contrast to the NaI: $\mathrm{Cl}^{-}$system, it shows only a small electric-field-induced shift, too small in fact for an accurate determination of the shifts. With $E_{\mathrm{dc}} \|[100]$, the observed shifts where $0.08 \pm 0.03$ $\mathrm{cm}^{-1}$ for $70-\mathrm{kV} / \mathrm{cm}$ applied and $E_{\mathrm{IR}} \| E_{\mathrm{dc}}$, and -0.05 $\pm 0.03 \mathrm{~cm}^{-1}$ for $75-\mathrm{kV} / \mathrm{cm}$ applied and $E_{\mathrm{IR}} \perp E_{\mathrm{dc}}$. The magnitudes of the shifts were too small to confirm the expected quadratic dependence on $E_{\mathrm{dc}}$, or to draw any conclusions about the field dependence of the linewidth or absorption strength. No measurements were made with $E_{\mathrm{dc}} \|[110]$.

$$
\text { C. } \mathrm{NaCl}: \mathrm{Cu}^{63} \mathrm{Cl}
$$

The ${ }^{63} \mathrm{Cu}^{+}$resonant-mode absorption in $\mathrm{NaCl}$ is at $23.57 \mathrm{~cm}^{-1} .^{2}$ Measurements were made with $E_{\mathrm{dc}} \|[100]$. With $E_{\mathrm{IR}} \| E_{\mathrm{dc}}$, applied fields of $120 \mathrm{kV} / \mathrm{cm}$ were reached; for $E_{\mathrm{IR}} \perp E_{\mathrm{dc}}$, up to $70 \mathrm{kV} / \mathrm{cm}$ was applied. In neither polarization was a shift of mode frequency of more than $0.005 \mathrm{~cm}^{-1}$ or a change in absorption strength of more than $5 \%$ detected. These limits reflect experimental precision.

\section{DISCUSSION OF RESULTS}

\section{A. $\mathrm{Nal}: \mathrm{NaCl}$}

\section{Theoretical Development}

In our analysis of the results, we first observe, as did Gebhardt, ${ }^{12}$ that the second-order Starkeffect operator has the same symmetry and (even) parity as does the uniaxial-stress operator. This

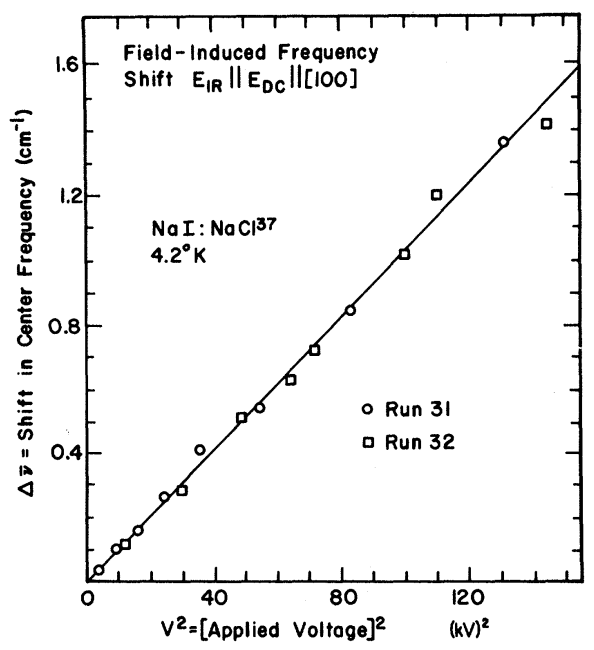

FIG. 7. Electric field shift data for $E_{\mathrm{dc}}\left\|[100] . \quad E_{\mathrm{IR}}\right\| E_{\mathrm{dc}}$. Data from two different runs on the same sample are shown. 
is because both operators are symmetric secondrank tensors. Specifically, the perturbation operator of the electric field has the form

$$
H_{e}=-\sum_{i=1}^{3} E x_{i} \zeta_{i}
$$

where $E$ is the local electric field strength and $\zeta_{i}$ are its direction cosines. For the second-order effect, the perturbation operator is

$$
H_{e}^{\prime}=H_{e} P H_{e}=\sum_{i, j} x_{i} \zeta_{i} E P x_{j} \zeta_{j} E
$$

where

$$
P=\sum_{\gamma} \frac{1}{E^{0}-E_{\gamma}^{0}}|\gamma\rangle\langle\gamma| .
$$

$E^{0}$ is the energy of the state to which the perturbation is applied and $|\gamma\rangle$ are the other unperturbed states of the system. The symmetry properties of $H_{e}^{\prime}$ are determined by the operator

$$
x_{i} x_{j} \quad(i, j=1,2,3),
$$

which is a symmetric second-rank tensor, since $x y-y x=0$. The stress tensor $\epsilon_{i j}$ is also a symmetric second-rank tensor. This leads to the same selection rules for both perturbations. We may now apply the considerable body of group-theoretical development devoted to the uniaxial-stress problem directly to the determination of the defect-site symmetry in our case.

In particular, the work of Kaplyanskii, ${ }^{13}$ as summarized by Fitchen, ${ }^{14}$ is most useful. Fitchen shows the intensity ratios for the split components of the zero-phonon line for all possible defect symmetries in cubic crystals, as a function of appliedstress direction (electric field direction in our

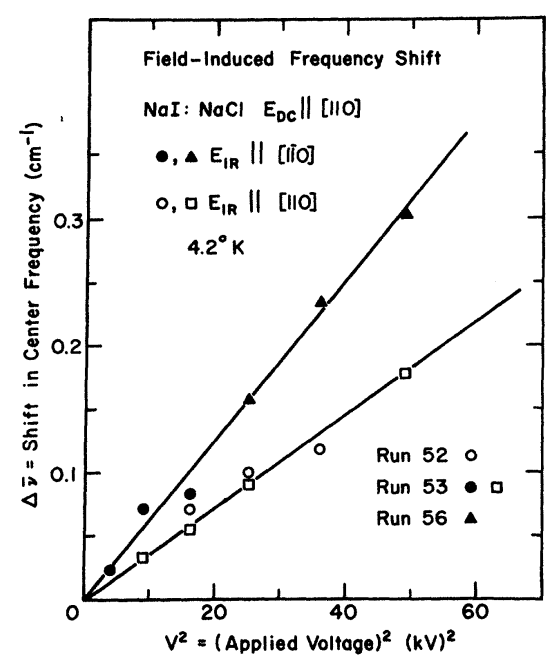

FIG. 8. Electric field shift data for $E_{\mathrm{dc}} \|[110]$. Two polarization directions for $E_{\mathrm{IR}}$ are shown. Data from three runs on the same sample are presented.

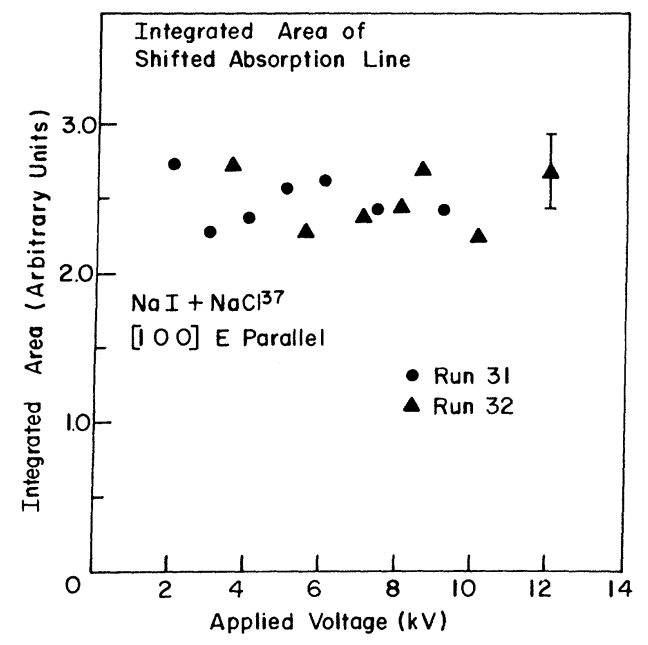

FIG. 9. Electric field dependence of the resonantmode strength. Data are for $E_{\mathrm{dc}} \|[100]$ and $E_{\mathrm{IR}} \| E_{\mathrm{dc}}$.

case) and polarization direction of the incident radiation. Our data on $\mathrm{NaI}: \mathrm{NaCl}$ for $E_{\mathrm{dc}}{ }^{\prime \prime}[100]$ show two distinct lines of equal intensity corresponding to the two polarization directions. For $E_{\mathrm{dc}} \|[110]$, we see three distinct lines of equal intensity for the three nonequivalent polarization directions.

By inspection of Fitchen's results, we find that only a cubic site with $A_{1} \rightarrow T_{1}$ unperturbed transition predicts the observed polarization dependences. Because the shift is quadratic with field, we may rule out those cubic sites which lack inversion symmetry. This leaves only $O_{h}$ and $T_{h}$ sites as candidates. We note that no site in an NaCl-type lattice has $T_{h}$ symmetry and conclude that the site symmetry is $O_{h}$ and that the observed transition is $A_{1 g} \rightarrow T_{1 u}$ in the unperturbed crystal.

By analogy with the uniaxial-stress development of Gebhardt, ${ }^{12}$ we may now also obtain a general expression for the shift $\hbar \Delta \Omega$ in transition energy with applied field. We first observe that the operator $x_{i}$ transforms like $T_{1 u}$ in $O_{h}$. The operator $x_{i} x_{j}$ then transforms like the symmetric product

$$
T_{1 u}^{2}=A_{1 g}+E_{g}+T_{2 g} \text {. }
$$

By applying the Wigner-Eckart theorem, Gebhardt shows that $\Delta \Omega$ may be written as a symmetric expression in the direction cosines $\zeta_{i}$ and $\eta_{i}$ :

$$
\begin{aligned}
\hbar \Delta \Omega / E^{2}= & A_{1}+A_{2}\left(2 \zeta_{3}^{2}-\zeta_{1}^{2}-\zeta_{2}^{2}\right)\left(2 \eta_{3}^{2}-\eta_{1}^{2}-\eta_{2}^{2}\right) \\
& +3 A_{3}\left(\zeta_{1}^{2}-\zeta_{2}^{2}\right)\left(\eta_{1}^{2}-\eta_{2}^{2}\right) \\
& +2 A_{5}\left(\zeta_{1} \zeta_{2} \eta_{1} \eta_{2}+\zeta_{2} \zeta_{3} \eta_{2} \eta_{3}+\zeta_{3} \zeta_{1} \eta_{3} \eta_{1}\right) .
\end{aligned}
$$

The $\zeta_{i}$ 's refer to the external dc field and the $\eta_{i}$ 's refer to the polarization of the incident radiation. The coefficients $A_{i}$ are identified with the $A_{1 g}$ (hydrostatic), $E_{g}$ (tetragonal), and $T_{2 g}$ (trigonal) com- 
ponents of the perturbation operator for $i=1,3,5$, respectively. They are analogous to strain-coupling coefficients $A, B$, and $C$ encountered in the uniaxial-stress problem. ${ }^{3}$

We now turn to a specific model in order to relate the frequency shifts to the details of the environment of the impurity ion. A particularly tractable approach is to let an anharmonic oscillator represent the impurity ion. Elliott et al. ${ }^{15,16}$ found this model well suited to describing many of the properties of the $U$ center in $\mathrm{CaF}_{2}$. In our case, because of the extremely low frequency of the resonant mode, the corresponding normal mode of the latticedefect system will consist of the $\mathrm{Cl}^{-}$ion moving $180^{\circ}$ out of phase with long-wavelength oscillations of the adjacent lattice. ${ }^{17}$ We can therefore describe the position of the $\mathrm{Cl}^{-}$ion in terms of its coordinates $(x, y, z)$ with respect to the center of mass of the surrounding lattice. Then an expansion of the potential energy of the impurity ion, consistent with the $O_{h}$-site symmetry already determined, gives

$$
\begin{aligned}
V_{0}(x, y, z)= & \frac{1}{2} m \omega^{2}\left(x^{2}+y^{2}+z^{2}\right)+\beta\left(x^{4}+y^{4}+z^{4}\right) \\
& +\gamma\left(x^{2} y^{2}+y^{2} z^{2}+z^{2} x^{2}\right)+O\left(x^{6}\right)+\cdots,
\end{aligned}
$$

where $m$ is the reduced mass. The isotope shift for resonant-mode systems is approximately the Einstein-oscillator value ${ }^{2}$

$$
\Omega_{1} / \Omega_{h}=\left(m_{h} / m_{1}\right)^{1 / 2},
$$

where $\Omega_{1}$ and $\Omega_{h}$ are the mode frequencies for isotopic impurities of mass $m_{1}$ and $m_{h}$, respectively. This indicates that the reduced masses for both the modes should be the impurity ion mass. This is

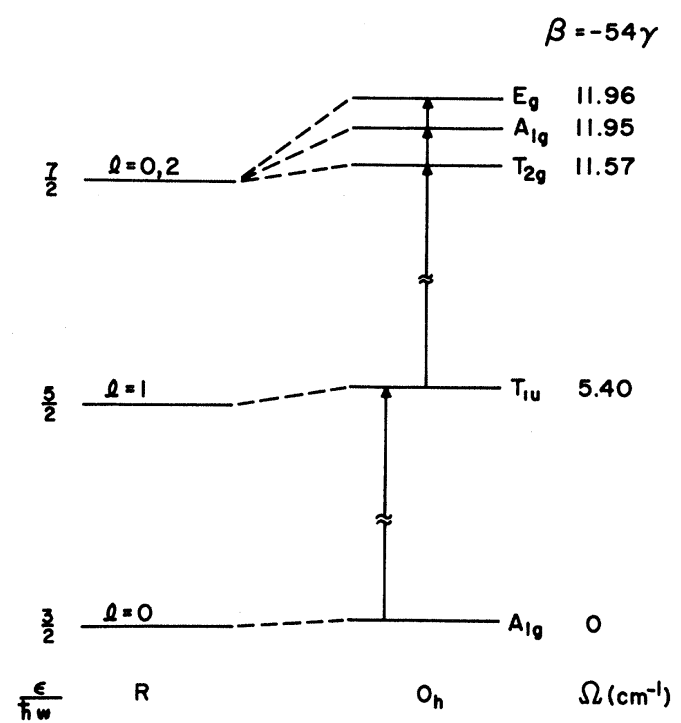

FIG. 10. Decomposition of the spherical-oscillator levels. Energies relative to the perturbed ground state are given, based on the parameters determined in the electric field measurements. consistent with our simple resonant picture of the $\mathrm{Cl}^{-}$ion moving out of phase with its neighbors; when a large number of neighbors are involved, their contribution to the reduced mass becomes negligible.

Figure 10 shows the decompostion of the three lowest spherical-oscillator states in an $O_{h}$ environment. Only the $l=2$ state has some of its degeneracy removed by the cubic crystal field. We identify the $5.4-\mathrm{cm}^{-1}$ resonant mode as the transition between the $A_{1 g}$ ground state and the $T_{1 u}$ first excited state. In terms of the model parameters, we obtain in Appendix A an expression for this transition energy

$$
\hbar \Omega=\hbar \omega+(3 \beta+\gamma)(\hbar / m \omega)^{2} .
$$

We also find the anharmonic-oscillator wave functions in terms of the linear-oscillator wave functions $|k \ln \rangle$ to the first order in $\beta$ and $\gamma$.

The effect of an applied electric field is the addition to $V_{0}$ of another term $H_{e}=-e \overrightarrow{\mathrm{E}} \cdot \overrightarrow{\mathrm{r}}$. This term is considered as a perturbation on the anharmonicoscillator wave functions already determined.

A second-order perturbation calculation (outlined in Appendix B) gives the shift of the ground state and the splittings of the first excited state for the field directions of high symmetry. These results are shown in Fig. 11. For $E_{\mathrm{dc}} \|[100]$, the $O_{h}$ symmetry is reduced to $C_{4 v}$ and the $T_{1 u}$ state splits into an $A_{1}$ and an $E$ state. For $E_{\mathrm{dc}} \|[110], C_{2 v}$ symmetry results and the $T_{1 u}$ state splits into an $A_{1}$, a $B_{1}$, and a $B_{2}$ state. The predicted changes $\hbar \Delta \Omega / E^{2}$ in the $O \rightarrow 1$ transition energy are listed in the fourth column of Table II in units of $\hbar e^{2} / 8 \mathrm{~m}^{3} \omega^{5}$.

We may now relate the coefficients $A_{i}$ to the model parameters $\omega, \beta$, and $\gamma$. Using the expressions given in Table II for the shifts, we find

$$
\begin{gathered}
A_{1}=16 \beta+\frac{16}{3} \gamma, A_{3}=8 \beta-\frac{4}{3} \gamma, A_{5}=-12 \beta+8 \gamma . \\
\text { 2. Comparison with Experiment }
\end{gathered}
$$

From the experimental values of the frequency shift given in Table I we can immediately determine the three coefficient $A_{i}$ 's. Equation (1) is evaluated for the two dc field directions studied and the results are listed in the third column of Table II.

Then we substitute the slopes from Table I for $\mathrm{NaI}: \mathrm{NaCl}$ into the appropriate expressions in Table II and find

$$
\begin{aligned}
& A_{1}=(6.4 \pm 0.2) \times 10^{-3} \mathrm{~cm}^{-1} /(\mathrm{kV} / \mathrm{cm})^{2}, \\
& A_{3}=(3.4 \pm 0.3) \times 10^{-3} \mathrm{~cm}^{-1} /(\mathrm{kV} / \mathrm{cm})^{2}, \\
& A_{5}=-(5.3 \pm 0.5) \times 10^{-3} \mathrm{~cm}^{-1} /(\mathrm{kV} / \mathrm{cm})^{2}
\end{aligned}
$$

In these expressions, the applied field (as opposed to the local field) is used. A similar calculation for the $E_{\mathrm{dc}} \|[100]$ data presented in Fig. 7 and Table I yields 


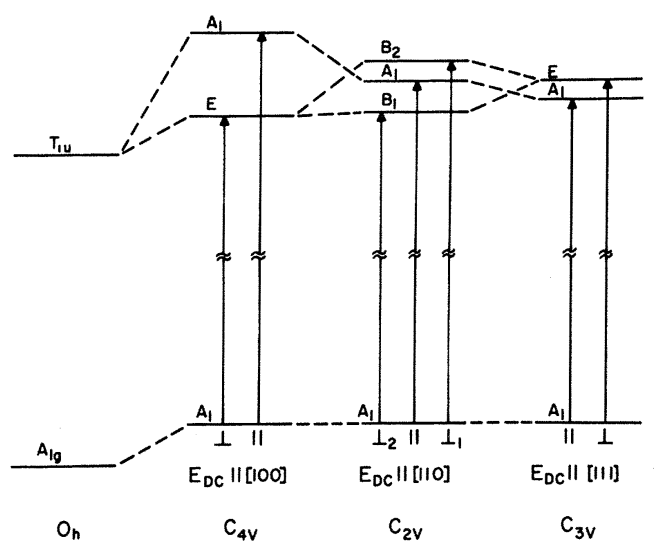

FIG. 11. Splitting and shift of the anharmonic-oscillator levels. Allowed transitions are indicated by solid lines for the three directions of high symmetry.

$$
\begin{aligned}
& A_{1}=(7.1 \pm 0.2) \times 10^{-3} \mathrm{~cm}^{-1} /(\mathrm{kV} / \mathrm{cm})^{2}, \\
& A_{3}=(3.3 \pm 0.1) \times 10^{-3} \mathrm{~cm}^{-1} /(\mathrm{kV} / \mathrm{cm})^{2} .
\end{aligned}
$$

The slight discrepancy in the $A_{1}$ values may be due to a dependence on isotopic mass and/or unperturbed mode frequency, since the [100] and [110] data were taken on different samples. In order to test this conjecture and, more importantly, in order to relate the frequency shifts to the details of the impurity's environment, we turn to our anharmonic-oscillator model.

We first check the internal consistency of our data with the model predictions. For the [110] field direction, the model predicts

$$
\frac{\Delta \Omega[110]-\Delta \Omega[001]}{\Delta \Omega[1 \overline{10}]}=\frac{18 \beta+8 \gamma-8 \gamma}{30 \beta}=0.60 \text {. }
$$

The experimentally determined slopes for the [110] sample, listed in Table I, give

$$
\begin{aligned}
\frac{\Delta \Omega[110]-\Delta \Omega[001]}{\Delta \Omega[1 \overline{1} 0]} & =\frac{(0.36 \pm 0.02)+(0.01 \pm 0.005)}{0.63 \pm 0.02} \\
& =0.59 \pm 0.04 .
\end{aligned}
$$

This close agreement indicates that the error incurred in dropping the higher-order terms in $\beta$, $\gamma$, and $(e E)^{2}$ in the perturbation calculation was negligible.

We may further check the consistency by comparing the $[100]$ data with predictions. The model predicts that

$$
\frac{\Delta \Omega[100]}{\Delta \Omega[1 \overline{10}]}=\frac{48 \beta}{30 \beta}=1.60 \text {. }
$$

Using the raw data in Table I we find

$$
\frac{\Delta \Omega[100]}{\Delta \Omega[1 \overline{1} 0]}=\frac{1.04 \pm 0.02}{0.63 \pm 0.02}=1.65 \pm 0.06 \text {. }
$$

The agreement, while not as good as seen above, is still valid within the experimental uncertainty.

However, we have seen that the model predicts a dependence of the shifts on isotopic mass and unperturbed mode frequency. Specifically, $\Delta \Omega$ $\sim\left(m^{3} \omega^{5}\right)^{-1}$. This correction is complicated by the concentration dependence of the mode frequency. ${ }^{11}$ To include this effect, we normalize the [100] data to the same $\mathrm{Cl}^{-}$concentration $(0.15 \%)$ as the [110] data. This gives 5.46 instead of $5.43 \mathrm{~cm}^{-1}$ for $\Omega[100]$. We approximate

$$
\frac{\omega[110]}{\omega[100]}=\frac{\Omega[110]}{\Omega[100]}
$$

because we have assumed the anharmonic contributions small throughout the calculation. The correction to Eq. (5) predicted by this approach is

$$
\left(\frac{5.54 \pm 0.02}{5.46 \pm 0.02}\right)^{5}\left(\frac{35.45}{37}\right)^{3}=0.96 \pm 0.04 \text {, }
$$

so that

$$
\frac{\Delta \Omega[100]}{\Delta \Omega[1 \overline{1} 0]}=(1.65 \pm 0.06)(0.96 \pm 0.04)=1.58 \pm 0.09 \text {. }
$$

Agreement with the predicted value of 1.6 is quite good.

We now desire quantitative determinations of the model parameters $\omega, \beta$, and $\gamma$ for the systems studied. In the case of $\mathrm{NaI}: \mathrm{NaCl}$, because of the high degree of internal consistency, we may use either the [100] or the [110] data to fit the model; we use the [100] data because the relative uncertainties are smaller.

Before proceeding further, however, we must relate the local field $E$ at the resonant-mode site to the applied field $E_{0}$. For a perfect crystal, the Lorentz correction has been shown ${ }^{18}$ to be valid:

$$
E=\frac{1}{3}(\epsilon+2) E_{0} \text {. }
$$

For a nonpolarizable impurity ion, the Onsager relation ${ }^{19}$ is believed to be appropriate:

$$
E=3 \epsilon /(2 \epsilon+1) E_{0} \text {. }
$$

In both these expressions, the dielectric constant is the one appropriate to the frequency region under consideration. Since resonant-mode frequencies

TABLE II. Calculated shifts of the absorption line for two field directions.

\begin{tabular}{lcrr}
\hline \hline $\begin{array}{l}\text { Direction } \\
\text { of } E_{\mathrm{dc}}\end{array}$ & $\begin{array}{l}\text { Direction } \\
\text { of polar- } \\
\text { ization } E_{\mathrm{IR}}\end{array}$ & $\begin{array}{c}\hbar \Delta \Omega / E^{2} \\
\left(\hbar e^{2} / 8 m^{3} \omega^{5}\right)\end{array}$ & $\begin{array}{r}\hbar \Delta \Omega / E^{2} \\
\left(\hbar e^{2} / 8 m^{3} \omega^{5}\right)\end{array}$ \\
\hline \hline$[100]$ & {$[100]$} & $A_{1}+4 A_{3}$ & $48 \beta$ \\
& {$[001]$} & $A_{1}-2 A_{3}$ & $8 \gamma$ \\
{$[110]$} & {$[110]$} & $A_{1}+A_{3}+\frac{1}{2} A_{5}$ & $18 \beta+8 \gamma$ \\
& {$[1 \overline{10} 0]$} & $A_{1}+A_{3}-\frac{1}{2} A_{5}$ & $30 \beta$ \\
& {$[001]$} & $A_{1}-2 A_{3}$ & $8 \gamma$ \\
\hline
\end{tabular}


are well below the resistrahl frequencies of the host lattices, the static dielectric constants are used herein.

Mahan $^{20}$ has shown that an approximate expression for the correction at an impurity site is

$$
E=\frac{1}{3}(\epsilon+2)\left[1-g \alpha\left(\alpha-\alpha^{\prime}\right)\right] E_{0},
$$

where $\alpha$ and $\alpha^{\prime}$ are the total (electronic +ionic) polarizabilities of the host and impurity ions, respectively, and $g$ represents a complicated wavevector sum which has not yet been evaluated. As expected, this reduces to the Lorentz correction for $\alpha=\alpha^{\prime}$.

We evaluate Eq. (6) in Appendix $\mathrm{C}$ and obtain $E=2.4 E_{0}$ for NaI: $\mathrm{NaCl}^{37}$. We now use this correction and the unperturbed impurity ion charge and mass to calculate the model parameters. By substituting the measured values of $\Omega$ and $\Delta \Omega(E)$ into Eq. (3) and the expressions for the frequency shift given in Table II, we obtain the values listed below:

$\beta=8.54 \times 10^{16} \mathrm{erg} / \mathrm{cm}^{4}, \quad \gamma=-0.16 \times 10^{16} \mathrm{erg} / \mathrm{cm}^{4}$,

$\omega=9.61 \times 10^{11} \mathrm{sec}^{-1}$.

The anharmonic contribution to the transition energy, calculated from Eq. (3), is $7 \%$. It is small, reconfirming the validity of the perturbation approach.

From the values listed above and Eq. (3), we can also predict the frequency shifts that result from isotopic mass substitutions. For NaI: $\mathrm{NaCl}$, the value obtained is

$$
\Omega(35) / \Omega(37)=1.030 \text {. }
$$

This is consistent with the value of $1.028 \pm 0.008$ reported by Clayman et al. ${ }^{11}$

Broadening of the absorption line with field is due to at least two contributions. Nonuniformity of the applied field strength, caused by fringing fields and surface imperfections, is probably the biggest contributor. Another important contribution arises from the decreased lifetime of the resonant mode as it shifts to higher frequencies. This is due to the higher density of states of host-lattice phonons. Another possible cause is nonuniformity of the local field strength. The extended nature of the resonant mode allows perturbation of the mode by local field nonuniformity at lattice sites somewhat removed from the $\mathrm{Cl}^{-}$impurity site. Such nonuniformity could arise from other lattice imperfections. The field-induced broadening has not been analyzed quantitatively.

\section{B. $\mathbf{K B r}: \mathbf{L i}^{7} \mathbf{B r}$}

The success of the anharmonic-oscillator model in explaining the $\mathrm{NaI}: \mathrm{NaCl}$ experimental results suggests that we try the same approach for
$\mathrm{KBr}: \mathrm{Li}^{7} \mathrm{Br}$. Fourth-order anharmonicity can cause the anomalously large ${ }^{6} \mathrm{Li}^{+}-{ }^{7} \mathrm{Li}^{+}$isotope shift in $\mathrm{KBr},{ }^{2}$ but the crucial test is whether or not the anharmonic-oscillator model can self-consistently explain both the isotope shift and the electric field shifts. We assume for the purpose of the test that the frequency shift is indeed quadratic.

With the measured resonant frequencies for ${ }^{6} \mathrm{Li}^{+}$ and ${ }^{7} \mathrm{Li}^{+}$in $\mathrm{KBr},{ }^{2}$ we can use Eq. (3) to obtain $\omega$ and $(3 \beta+\gamma)$, finding

$$
\begin{aligned}
\omega\left(\mathrm{Li}^{7}\right) & =22.0 \times 10^{11} \mathrm{sec}^{-1}=12 \mathrm{~cm}^{-1}, \\
(3 \beta+\gamma) & =52 \times 10^{16} \mathrm{ergs} / \mathrm{cm}^{4},
\end{aligned}
$$

so that the quartic perturbation must be very large $(\sim 33 \%)$ to explain the isotope shift. This result is consistent with the one-dimensional calculation

(37\%) described in Ref. 2.

In Table II, expressions for the observed electric field shifts are given in terms of the oscillator parameters $\beta, \gamma$, and $\omega$. Making the Lorentz localfield correction to overestimate the local field, we find for $\mathrm{KBr}: \mathrm{Li}^{7} \mathrm{Br}$

$$
\begin{aligned}
3 \beta & =(1.1 \pm 0.4) \times 10^{16} \mathrm{ergs} / \mathrm{cm}^{4}, \\
\gamma & =-(1.2 \pm 0.7) \times 10^{16} \mathrm{ergs} / \mathrm{cm}^{4},
\end{aligned}
$$

so that

$$
(3 \beta+\gamma)=(-0.1 \pm 0.8) \times 10^{16} \mathrm{ergs} / \mathrm{cm}^{4} .
$$

This result shows that the amount of anharmonicity necessary to explain the isotope shift is greater by at least a factor of 50 than that required to explain the electric field shifts. Furthermore, the value of $(3 \beta+\gamma)$ calculated from the electric field shifts may in fact be negative, which would make the isotope shift smaller than that of a harmonic oscillator. Thus, we must look elsewhere if we are to understand both sets of experimental results.

Another mechanism which could be important is a third-order dynamic coupling of the resonantmode oscillator to the phonons. It has been shown ${ }^{5}$ that such an interaction will cause a small negative shift of the resonant-mode line for $E_{\mathrm{IR}}\left\|E_{\mathrm{dc}}\right\|[100]$. However, this anharmonic interaction also reduces the isotope shift from that of an Einstein oscillator, so that no combination of fourth-order static anharmonicity and third-order dynamic coupling can explain both the isotope shift and the electric field shifts.

Recently, Quigley and $\operatorname{Das}^{21}$ and Wilson et al. ${ }^{22}$ have performed calculations to determine the minimum energy configuration of $\mathrm{Li}^{+}$in $\mathrm{KBr}$. Their results indicate that the potential-energy minima for the impurity ion are displaced in the [111] directions from the center of the host-ion $\left(\mathrm{K}^{+}\right)$cavity, as has been confirmed for $\mathrm{Li}^{+}$in $\mathrm{KCl}$. The barriers on the [110] directions between adjacent equilibrium sites are small; Quigley and Das find that they are 
smaller than the $\mathrm{Li}^{*}$ vibrational zero-point energy. In this case, we should consider the $\mathrm{Li}^{+}$ion as oncenter in a very anharmonic well. Gomez ${ }^{23}$ has pointed out that such a well can explain the large ${ }^{6} \mathrm{Li}^{+}-{ }^{7} \mathrm{Li}^{+}$isotope shift in $\mathrm{KBr}$. The question remains whether this type of well gives a small Stark effect; we now investigate this possibility.

Svare $^{24}$ recently proposed a model in which the low [110] barriers are removed entirely. This leaves the $\mathrm{Li}^{+}$ion free to move in curve equipotential "tubes" in the [110] planes. The motion in each tube is that of a particle in a one-dimensional box, with energy levels given by

$$
E_{n}=\left(\pi^{2} \hbar^{2} / 2 m L^{2}\right) n^{2}, \quad n=1,2, \cdots .
$$

Svare finds that $L \approx 2 \AA$ fits the experimentally determined $\mathrm{KBr}$ : $\mathrm{LiBr}$ absorption frequencies and is consistent with the calculations of Quigley and Das and Wilson et al.

The Stark-effect transition-energy shift of a particle in a one-dimensional box is given by

$$
\hbar \Delta \Omega=\left(4 L^{2} e^{2} / \hbar \Omega_{0} \pi^{4}\right) E^{2}
$$

for the ground-state to first-excited-state transition. Using the Lorentz local-field correction for an applied field of $70 \mathrm{kV} / \mathrm{cm}$, we find $\hbar \Delta \Omega=1.6$ $\mathrm{cm}^{-1}$. This is in marked disagreement with the measured value of $0.08 \pm 0.03 \mathrm{~cm}^{-1}$. Even if one were to use the applied field in the calculation, $\hbar \Delta \Omega$ lies a factor of 3 above the observed value. We conclude that the extreme anharmonicity represented by the square well is not appropriate to the $\mathrm{KBr}: \mathrm{LiBr}$ system. The same conclusion was reached earlier by Alexander et al. ${ }^{3}$; they noted marked discrepancies between the observed temperature dependence and that predicted by Svare's model.

A more fruitful approach is to introduce the central barrier as a perturbation to a harmonic well. We consider a one-dimensional harmonic oscillator with a perturbing potential of the form

$$
H_{p}=A e^{-b x^{2}},
$$

so that the Hamiltonian becomes

$$
H=P^{2} / 2 M+\frac{1}{2} M \omega^{2} x^{2}+A e^{-b x^{2}} .
$$

Treating $H_{p}$ as a perturbation, the first-order perturbed eigenvalues are easily shown to be

$$
\begin{aligned}
& E_{0}=\frac{1}{2} \hbar \omega+A R^{1 / 2} \quad \text { (ground state), } \\
& E_{1}=\frac{3}{2} \hbar \omega+A R^{3 / 2} \quad \text { (first excited state), }
\end{aligned}
$$

where $R=M \omega /(b \hbar+M \omega)$.

The experimental resonant frequencies $\Omega\left({ }^{7} \mathrm{Li}^{+}\right)$ $=16.07 \mathrm{~cm}^{-1}$ amd $\Omega\left({ }^{6} \mathrm{LI}^{+}\right)=17.7 \mathrm{~cm}^{-1}$ can be fitted with a range of the model parameters $A, b$, and $\omega$. One particular choice which gives the correct isotope shift is $A=11.7 \mathrm{~cm}^{-1}, b=1.19 \times 10^{17} \mathrm{~cm}^{-2}$, and

$\omega\left({ }^{7} \mathrm{Li}^{+}\right)=20.5 \mathrm{~cm}^{-1} . \quad \omega\left({ }^{6} \mathrm{Li}^{+}\right)$is then given by

$$
\omega\left({ }^{6} \mathrm{Li}^{+}\right)=\frac{7}{6} \omega\left({ }^{7} \mathrm{Li}^{+}\right)=22.14 \mathrm{~cm}^{-1} \text {. }
$$

These parameter values change the $O \rightarrow 1$ transition energy by $-22 \%$. In spite of the fact that this is a rather large perturbation, the second-order energy corrections (which can be summed exactly) are negligible, since they change the transition energies by only $0.08 \mathrm{~cm}^{-1}$. Their effect on the isotope shift is found to be less than $0.05 \%$, which is well inside the experimental uncertainty.

The solid curve in Fig. 12(a) shows the unperturbed potential well $\frac{1}{2} M \omega^{2} \times x^{2}$ and the ground- and first-excited-state energy levels. The dash curve shows $H_{p}$ for our choice of parameters. Figure 12(b) shows the resulting perturbed potential well and the perturbed ground- and first-excited-state energy levels. As can be seen, the height of the central potential-energy barrier is considerably less than the ground-state energy.

We now introduce the electric field perturbation $H_{e}=-e E x$. Using the first-order perturbed eigenfunctions of Eq. (10), we can calculate the electricfield-induced shift in the $O \rightarrow 1$ transition energy. Making the Lorentz local-field correction, the shift is found to be $0.03 \mathrm{~cm}^{-1}$ for an external field of $70 \mathrm{kV} / \mathrm{cm}$. This value is comparable to the observed shift of $0.08 \pm 0.03 \mathrm{~cm}^{-1}$. We see then that a static potential well which has displaced energy minima, but barriers which are small compared to the impurity zero-point energy, causes a large isotope shift, but only a small electric shift. The discrepancy between the calculated field shift and that observed experimentally could be eliminated by a small increase in the $x^{4}$ component of the perturbation potential, with a negligible effect on the calculated isotope shift. The important point
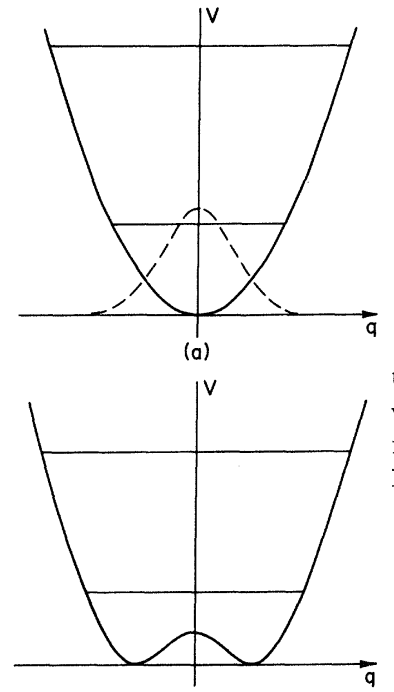

(b)
FIG. 12. One-dimensional "off-center" model. (a) Harmonic well with two lowest states. Dash curve shows perturbing potential used. (b) Anharmonic well with two lowest states. Parameters are appropriate for $\mathrm{KBr}: \mathrm{Li}^{+}$. 
here is that a static potential well of a form for which there is some theoretical justification explains the available experimental results on $\mathrm{KBr}: \mathrm{LiBr}$.

$$
\text { C. } \mathrm{NaCl}: \mathrm{Cu}^{63} \mathrm{Cl}
$$

As we have seen, the electric-field-induced shifts due to fourth-order anharmonicity vary as $m^{-3} \omega^{-5} . \mathrm{NaCl}: \mathrm{Cu}^{63} \mathrm{Cl}$ has both a large impurity mass and a high resonant frequency, thus the calculated shifts would be only $\sim 10^{-4}$ as large as the $\mathrm{NaI}: \mathrm{NaCl}$ shifts. A shift this small would be unobservable.

The main impetus for studying $\mathrm{NaCl}: \mathrm{Cu}^{+}$is that minimum energy calculations by Wilson et al. ${ }^{22}$ predict that $\mathrm{Cu}^{+}$should be off-center in the [111] directions, with an associated permanent electric dipole moment. If so, and if the tunneling barrier are large compared to the impurity zero-point energy, there would be closely-spaced tunneling levels which split in the presence of an external electric field. This would be reflected in a splitting or broadening of the observed resonant-mode absorption line at $23.57 \mathrm{~cm}^{-1}$, or in a change in absorption strength. The experimental results on $\mathrm{NaCl}: \mathrm{Cu}^{63} \mathrm{Cl}$ show that no shift or broadening in excess of $0.005 \mathrm{~cm}^{-1}$ occurs, putting an upper limit of $\sim 10^{-3} e A$ (uncorrected for local fields) on the impurity dipole moment. In contrast, the $\mathrm{Li}^{+}$dipole moment in $\mathrm{KCl}$ is $\sim 1.2 \mathrm{eA} .^{1}$ Although a potential similar to that which explains the $\mathrm{KBr}: \mathrm{Li}^{+}$results can be used here, $\mathrm{Cu}^{+}$is still effectively on-center.

\section{SUMMARY}

For $\mathrm{NaI}: \mathrm{NaCl}$, the resonant-mode frequency shifted quadratically with applied electric field. The magnitudes and polarization dependences of the shifts were found to be totally consistent with an "on-center" substitutional site for the $\mathrm{Cl}^{-}$impurity. A three-dimensional harmonic oscillator perturbed simultaneously by the cubic crystal field (to fourthorder in the ionic displacements) and an external electric field was found to be in perfect agreement with the relative magnitudes of the shifts for different polarization directions. Using an approximate local-field correction, all model parameters could be determined, and were also found to be in agreement with previously determined isotopeshift measurements.

For $\mathrm{KBr}: \mathrm{LiBr}$, small shifts of mode frequency with field were measured. It was found that the simple-oscillator model with quartic anharmonicity could not be consistently applied to both the Starkeffect and isotope-shift results. A one-dimensional model, in which the potential perturbing the harmonic oscillator was a small "bump" at the center of the well, was found to give semiquantitative agreement with both the large isotope shift and the small Stark effect. Because the central barrier was less than the zero-point energy of the oscillator, the impurity ion still appeared "on-center."

In the case of $\mathrm{NaCl}: \mathrm{CuCl}$, no shift of mode frequency, line broadening, or change in absorption strength with field was observed. This null result is consistent with an "on-center" resonant-mode configuration for this system; it is contrary to recent theoretical predictions.

We have seen that externally applied electric fields are useful probes of resonant-mode systems. They can provide conclusive evidence of impuritysite symmetry and can differentiate between onand off-center configurations. For two of the defect systems studied, we have been able to establish some definite features of the potentials associated with low-lying modes. For $\mathrm{Cl}^{-}$in NaI, the potential is approaching that of a square well, while for the much smaller $\mathrm{Li}^{+}$ion in $\mathrm{KBr}$, a central instability does already occur. Uncertainty about the local electric field at the impurity site is the major difficulty in obtaining quantitative results. This situation is analogous to the uncertainty about the local elastic constants in the case of uniaxial-stress perturbations. ${ }^{3}$ In both cases, detailed analysis in terms of realistic models awaits further work on the determination of the local fields.

\section{ACKNOWLEDGMENTS}

We wish to acknowledge helpful discussions with Dr. R. O. Pohl, Dr. J. B. Page, Dr. A. E. Hughes, and Dr. A. S. Barker, Jr.

\section{APPENDIX A: ANHARMONIC OSCILLATOR}

The starting point of the perturbation calculation is the isotropic harmonic oscillator

$$
H_{h}=|P|^{2} / 2 m+\frac{1}{2} m \omega^{2}\left(x^{2}+y^{2}+z^{2}\right) .
$$

The wave functions $\left(\left|\psi_{s}^{0}\right\rangle\right.$, where $\left.s=0,1,2, \ldots\right)$ can be written as the superposition of three linear oscillators

$$
\left|\psi_{s}^{0}\right\rangle=|k \ln \rangle ; \quad E_{s}^{0}=\hbar \omega\left(s+\frac{3}{2}\right)
$$

where $s=k+l+n . k, l$, and $n$ can be considered the quantum numbers of three linear oscillators in the $x, y$, and $z$ directions, respectively.

At a substitutional lattice site, the oscillator will see a potential well of cubic $\left(O_{h}\right)$ symmetry. In this case, an expansion of the potential gives additional quartic terms. These are "anharmonic" contributions

$$
H_{a}=\beta\left(x^{4}+y^{4}+z^{4}\right)+\gamma\left(x^{2} y^{2}+y^{2} z^{2}+z^{2} x^{2}\right) .
$$

We treat $H_{a}$ as a perturbation of the harmonic oscillator. We will find that some of the degenerate levels are split. By symmetry arguments alone one obtains the representations of the oscillator wave functions for the isotropic and the cubic cases. 
These are given in Fig. 10 for the three lowestlying states. The cubic field splits the $s+d$ degenerate level into $E_{g}, A_{1 g}$, and $T_{2 g}$ levels. To relate the shifts and splittings of the energy levels to the model parameters $\omega, \beta$, and $\gamma$, we seek solutions of

$$
\left(H_{h}+H_{a}\right)\left|\psi_{s}\right\rangle=E_{s}\left|\psi_{s}\right\rangle
$$

to first order in $\beta$ and $\gamma$. We write the perturbed eigenfunctions as

$$
\left|\psi_{s}\right\rangle=\left|\psi_{s}^{0}\right\rangle+\left|\psi_{s}^{\prime}\right\rangle,
$$

and the eigenvalues as

$$
E_{s}=E_{s}^{(0)}+E_{s}^{(1)} \text {. }
$$

Because the unperturbed excited states $\left|\psi_{s}^{0}\right\rangle(s$ $=1,2, \cdots)$ are degenerate, we must solve a $3 \times 3$ matrix for $s=1$ and a $6 \times 6$ matrix for $s=2$.

All of the matrix elements $V_{i j}=\left\langle i\left|H_{a}\right| j\right\rangle$ for $s$ $=0,1,2$ are given in Table III below in units of $(\hbar / 2 m \omega)^{2}$. The higher states will not be considered because they do not enter the later calculations. The designation of the basis states for each value of $s$ is also given.

The matrix is diagonal for $s=1$ and produces the expected threefold degenerate $\left(T_{1 u}\right)$ state. For $s=2$, the $6 \times 6$ matrix factors into a diagonal $3 \times 3$ and a symmetric nondiagonal $3 \times 3$. The diagonal $3 \times 3$ yields the expected $T_{2 g}$ state and the other $3 \times 3$ produces the $A_{1 g}$ and the $E_{g}$ states. These results are summarized in Table IV. We give the perturbed eigenstates (to zero order in $\beta$ and $\gamma$ ), their symmetry, and the parameters $d_{i}$ which characterize the perturbation to the harmonic-oscillator energy levels:

$$
E_{s}^{(1)}=\left(d_{1} \beta+d_{2} \gamma\right)(\hbar / 2 m \omega)^{2} .
$$

The change in transition energy between levels $s$ and $t$ is

$$
\hbar \Omega_{s t}=E_{t}^{(1)}-E_{s}^{(1)}
$$

TABLE III. Matrix Elements for $V_{i j}=\left\langle i\left|H_{a}\right| j\right\rangle$ for $s=0,1,2$ in units of $(\hbar / 2 m w)^{2}$.

\begin{tabular}{ccc}
\hline \hline$s$ & Matrix element & Basis designation \\
\hline 0 & $V_{11}=9 \beta+3 \gamma$ & $|1\rangle=|000\rangle$ \\
1 & $V_{11}=21 \beta+7 \gamma$ & $|1\rangle=|100\rangle$ \\
& $V_{22}=V_{33}=V_{11}$ & $|2\rangle=|010\rangle$ \\
& $V_{i j}=0$, otherwise & $|3\rangle=|001\rangle$ \\
2 & $V_{11}=33 \beta+15 \gamma$ & $|1\rangle=|110\rangle$ \\
& $V_{22}=V_{33}=V_{11}$ & $|2\rangle=|011\rangle$ \\
& $V_{44}=45 \beta+11 \gamma$ & $|3\rangle=|101\rangle$ \\
& $V_{55}=V_{66}=V_{44}$ & $|4\rangle=|200\rangle$ \\
& $V_{45}=V_{56}=V_{64}=2 \gamma$ & $|5\rangle=|020\rangle$ \\
& $V_{54}=V_{65}=V_{46}=2 \gamma$ & $|6\rangle=|002\rangle$ \\
& $V_{i j}=0$, otherwise & \\
\hline
\end{tabular}

TABLE IV. Properties of the anharmonic oscillator.

\begin{tabular}{lclrr}
\hline \hline$s$ & Symmetry & Zero-order eigenstate & $d_{1}$ & $d_{2}$ \\
\hline 0 & $A_{1 \boldsymbol{g}}$ & $|000\rangle$ & 9 & 3 \\
1 & $T_{1 u}$ & $|001\rangle$, etc. & 21 & 7 \\
2 & $A_{1 \boldsymbol{g}}$ & $(3)^{-1 / 2}(|200\rangle+|020\rangle$ & & \\
& & $+|002\rangle\rangle$ & 45 & 15 \\
2 & $E_{\boldsymbol{g}}$ & $(2)^{-1 / 2}(|200\rangle-|020\rangle)$ & & \\
& & $(6)^{-1 / 2}(|200\rangle+|020\rangle$ & & \\
& & $-2|002\rangle)$ & 45 & 9 \\
2 & $T_{2 g}$ & $|011\rangle$, etc. & 33 & 15 \\
& & & \\
\hline
\end{tabular}

For example,

$$
\begin{aligned}
& \hbar \Omega_{01}=[(21 \beta+7 \gamma)-(9 \beta+3 \gamma)](\hbar / 2 m \omega)^{2}, \\
& \hbar \Omega_{01}=(3 \beta+\gamma)(\hbar / m \omega)^{2} .
\end{aligned}
$$

This is the first-order correction to the $O \rightarrow 1$ transition energy and appears as the anharmonic term in Eq. (3).

We will need the perturbed eigenstates to first order in $\beta$ and $\gamma$ in the later calculations. These are easily found as linear combinations of the harmonic-oscillator states:

$$
\left|\psi_{s}^{1}\right\rangle=\sum_{t \neq s, s^{\prime}} \frac{\left\langle\psi_{t}^{0}\left|H_{a}\right| \psi_{s}^{0}\right\rangle}{E_{s}^{(0)}-E_{t}^{(0)}}\left|\psi_{t}^{0}\right\rangle+\sum_{s^{\prime} \neq s} a_{s^{\prime}} \mid \psi_{s^{\prime}}^{0},
$$

where

$$
a_{s^{\prime}}=\frac{1}{E_{s}^{(1)}-E_{s^{\prime}}^{(1)}} \sum_{m \neq s, s^{\prime}} \frac{\left\langle\psi_{s^{\prime}}^{0}\left|H_{a}\right| \psi_{m}^{0}\right\rangle\left\langle\psi_{m}^{0}\left|H_{a}\right| \psi_{s}^{0}\right\rangle}{E_{s}^{(0)}-E_{m}^{(0)}} .
$$

$\left|\psi_{s^{\prime}}^{0}\right\rangle$ are the states with which $\left|\psi_{s}^{0}\right\rangle$ is degenerate. We find for $s=1$ and 2 that $a_{s^{\prime}}=0$ for all $s^{\prime}$. The perturbed wave functions are given below. For $s$ $=0$, the complete expression is given to first order $\beta$ and $\gamma$. For $s=1$ and $s=2$, only those terms needed later in the electric field calculation are given.

$$
\begin{aligned}
& s=0: \mid\left.\psi\left(A_{1 g}\right)\right\rangle=|000\rangle-(3 \beta+\gamma)(\sqrt{2} / \hbar \omega)(\hbar / 2 m \omega)^{2} \\
& \times(|002\rangle+|020\rangle+|200\rangle) \\
&-6 \beta(\sqrt{2} / \hbar \omega)(\hbar / 2 m \omega)^{2}(|004\rangle+|040\rangle+|400\rangle) \\
&-(1 / \hbar \omega)(\hbar / 2 m \omega)^{2}(|220\rangle+|022\rangle+|202\rangle), \\
& s=1: \quad\left|\psi_{3}\left(T_{1 u}\right)\right\rangle=|001\rangle-(5 \beta+\gamma)(\sqrt{6} / \hbar \omega)(\hbar / 2 m \omega)^{2} \\
& \quad \times|003\rangle-(3 \beta+2 \gamma)(\sqrt{2} / \hbar \omega)(\hbar / 2 m \omega)^{2}(|021\rangle \\
&+|201\rangle)+\cdots, \\
& s=2:\left|\psi\left(A_{1 g}\right)\right\rangle=(|200\rangle+|020\rangle+|002\rangle) / \sqrt{3} \\
&+(9 \beta+3 \gamma)(\sqrt{2} / \hbar \omega)(\hbar / 2 m \omega)^{2} \frac{|000\rangle}{\sqrt{3}}+\cdots, \\
& s=2:\left|\psi_{1}\left(E_{g}\right)\right\rangle=(|200\rangle-|020\rangle) / \sqrt{2}+\cdots ; \\
&\left|\psi_{2}\left(E_{g}\right)\right\rangle=(|200\rangle+|020\rangle-2|002\rangle) / \sqrt{6}+\cdots
\end{aligned}
$$


TABLE V. Results of the electric field perturbation calculation.

\begin{tabular}{llccc}
\hline $\begin{array}{c}\text { Polari- } \\
\text { zation }\end{array}$ & $\begin{array}{c}\text { Sym- } \\
\text { metry }\end{array}$ & Mode & $\begin{array}{c}\hbar \Delta \Omega \\
\hbar(e E)^{2} / 8 m^{3} \omega^{5}\end{array}$ & $\begin{array}{c}E_{\mathrm{IR}} \text { polar- } \\
\text { ization }\end{array}$ \\
\hline \multirow{2}{*}[100]{} & $C_{4 v}$ & $A_{1}$ & $48 \beta$ & {$[100]$} \\
& & $A_{1}$ & $18 \beta+8 \gamma$ & $(100)$ \\
{$[110]$} & $C_{2 v}$ & $B_{1}$ & $30 \beta$ & {$[110]$} \\
& & $B_{2}$ & $8 \gamma$ & {$[110]$} \\
& & $A_{1}$ & $8 \beta+\frac{32}{3} \gamma$ & {$[001]$} \\
{$[111]$} & $C_{3 v}$ & $E$ & $20 \beta+\frac{8}{3} \gamma$ & {$[111]$} \\
& & & & \\
\hline \hline
\end{tabular}

$$
s=2:\left|\psi_{3}\left(T_{2 g}\right)\right\rangle=|011\rangle+\cdots, \text { etc. }
$$

\section{APPENDIX B: STARK EFFECT}

We now introduce the externally applied electric field $E$, considering it as a perturbation to the two lowest-lying states of the anharmonic oscillator:

$$
H_{e}=-e E\left(\zeta_{1} x+\zeta_{2} y+\zeta_{3} z\right),
$$

where the $\zeta_{i}$ 's are the three direction cosines of $E$ with respect to the crystal axes. The first-order change in energy is zero,

$$
E_{s}^{(1)}=\left\langle\psi_{s}\left|H_{e}\right| \psi_{s}\right\rangle=0 \text { for all } s .
$$

In second order, the perturbation operator becomes $^{25}$

$$
H_{e}^{\prime}=H_{e} P_{s} H_{e},
$$

where

$$
P_{s}=\sum_{t \neq s} \frac{\left|\psi_{t}\right\rangle\left\langle\psi_{t}\right|}{E_{s}-E_{t}}
$$

The ground state is a singlet so that

$$
E_{0}^{(2)}=\left\langle\psi_{0}\left|H_{e}^{\prime}\right| \psi_{0}\right\rangle=(24 \beta+8 \gamma) \hbar(e E)^{2} / 8 m^{3} \omega^{5} .
$$

Here, as in all similar expressions to follow, we drop all terms of zero order in $\beta$ or $\gamma$ because of a well-known property of a harmonic oscillator: There is no shift in transition energy under an applied electric field. We retain only terms of first order in $E^{2}, \beta$, and $\gamma$.

The first excited state is degenerate, so we must solve a $(3 \times 3)$ determinant to obtain the eigenvalues $E_{1}^{(2)}$ :

$$
\left|\left\langle\psi_{s}\left|H_{e}^{\prime}\right| \psi_{s^{\prime}}\right\rangle-E_{1}^{(2)} \delta_{s s^{\prime}}\right| \equiv\left|P_{s s^{\prime}}-E_{1}^{(2)} \delta_{s s^{\prime}}\right|=0 .
$$

Here $s$ and $s^{\prime}$ run over the three $T_{1 u}$ states. For general values of $\zeta_{1}, \zeta_{2}$, and $\zeta_{3}$, the secular equation has no simple form.

We may, however, write down the matrix elements $P_{s s^{\prime}}$ for the general case using the basis states $\left|\psi_{i}\left(T_{1 u}\right)\right\rangle$, where $i=1,2,3$ :

$$
P_{11}=\zeta_{1}^{2}\left[-(e E)^{2} / 2 m \omega^{2}+(72 \beta+8 \gamma) \hbar(e E)^{2} / 8 m^{3} \omega^{5}\right]
$$

$$
\begin{aligned}
& +\left(\zeta_{2}^{2}+\zeta_{3}^{2}\right)\left[-(e E)^{2} / 2 m \omega^{3}+(24 \beta+16 \gamma) \hbar(e E)^{2} / 8 m^{3} \omega^{5}\right], \\
& P_{12}=\zeta_{1} \zeta_{2}(-12 \beta+8 \gamma) \hbar(e E)^{2} / 8 m^{3} \omega^{5}=P_{21} .
\end{aligned}
$$

The other matrix elements are obtained by cyclic permutations of the subscripts.

The perturbation matrix simplifies considerably when the external electric field is applied along directions of high crystal symmetry. The results of the calculations for these cases are summarized in Table V. Listed are the direction of the dc field and the corresponding reduced-site symmetry. Also listed are the irreducible representation which each level transforms like, the change in transition en$\operatorname{ergy} \hbar \Delta \Omega$ between the $A_{1}$ ground state and the level, and the polarization direction corresponding to an allowed transition. Note that in the case of the degenerate levels, we specify the plane in which the incident radiation must be polarized for an allowed transition.

These results are also shown in Fig. 11 for $\beta$ $=-10 \gamma$. Allowed transitions are indicated by solid lines. For the case of $E_{\mathrm{dc}} \|[110], \perp_{1}$ refers to $E_{\mathrm{IR}} \|[1 \overline{1} 0]$ and $\perp_{2}$ refers to $E_{\mathrm{IR}} \|[001]$.

\section{APPENDIX C: APPROXIMATE LOCAL-FIELD CORRECTION}

Mahan ${ }^{20}$ relates the applied field $E_{0}$ to the local field $E$ by

$$
E=\frac{1}{3} \epsilon+2\left[1-g \alpha\left(\alpha-\alpha^{\prime}\right)\right] E_{0},
$$

where $\alpha$ and $\alpha^{\prime}$ are the polarizabilities of the host and substitutional impurity ion, respectively. At far-infrared frequencies, the static dielectric constant $\epsilon_{s}=6.60$ for NaI is used. To evaluate Eq. (6), we make three assumptions. (i) The Onsager ${ }^{19}$ relation holds when $\alpha^{\prime}=0$ :

$$
\frac{1}{3}(\epsilon+2)\left(1-g \alpha^{2}\right) E_{0}=[3 \epsilon /(2 \epsilon+1)] E_{0} .
$$

(ii) The ionic polarizability of the resonant mode is

$$
\alpha_{\text {ionic }}^{\prime}=(\mu / m)\left(\omega_{R} / \Omega\right)^{2}(f)\left(\alpha_{\text {NaI }}\right)_{\text {ionic }} \text {. }
$$

Here $\omega_{R}$ is the frequency of the host-lattice TO mode at $k=0, \mu$ is the NaI reduced mass, $m$ is the $\mathrm{Cl}^{-}$mass, and $f$ is the oscillator strength of the resonant mode. This is based on a comparison with the reststrahl process in the unperturbed NaI crystal. (iii) The electronic polarizability of the resonant mode stems from two equally weighted contributions:

$$
\alpha_{\mathrm{elec}}^{\prime}=\left(\alpha_{\mathrm{C} 1^{-}}\right)_{\mathrm{elec}}+\frac{1}{2}\left(\alpha_{\mathrm{Na}}+\alpha_{\mathrm{I}-}\right)_{\mathrm{elec}} .
$$

The required polarizabilities are listed in Table VI. $\alpha_{\text {ionic }}$ is assumed to be equal to $\alpha_{\text {T OT }}-\alpha_{\text {elec }}{ }^{18,26}$

Using these polarizabilities and the experimental results for $\mathrm{NaI}: \mathrm{NaCl}$ we find $E$. We first empirically evaluate the constant $g$ using the Onsager relation and assumption (i) above: 
TABLE VI. Electronic, ${ }^{\mathrm{a}}$ ionic, and total polarizability ${ }^{b}$ of $\mathrm{Na}^{+}, \mathrm{I}^{-}, \mathrm{Cl}^{-}$ions.

\begin{tabular}{lllr}
\hline \hline Ion & $\begin{array}{l}\alpha_{\text {elec }} \\
\left(\AA^{3}\right)\end{array}$ & $\begin{array}{c}\alpha_{\text {ionic }} \\
\left(\AA^{3}\right)\end{array}$ & $\begin{array}{c}\alpha_{\text {TOT }} \\
\left(\AA^{3}\right)\end{array}$ \\
\hline $\mathrm{Na}^{+}$ & 0.255 & 1.35 & 1.61 \\
$\mathrm{I}^{-}$ & 6.20 & 2.51 & 8.71 \\
$\mathrm{Cl}^{-}$ & 2.98 & 2.00 & 4.98 \\
\hline \hline
\end{tabular}

${ }^{a}$ Values taken from Tessman et al. (Ref. 18).

${ }^{\text {b }}$ Values taken from Roberts (Ref. 26).

$$
\frac{1}{3}\left(\epsilon_{s}+2\right)\left(1-g \alpha^{2}\right)=\frac{3 \epsilon_{s}}{2 \epsilon_{s}+1}=\frac{3(6.60)}{2(6.60)+1}=1.40,
$$

*Research supported mainly by the U. S. Atomic Energy Commission under Contract No. AT (30-1)-2391, Technical Report No. NYO-2391-119. Additional support was received from the Advanced Research Projects Agency through space and technical facilities of the Materials Science Center at Cornell University, MSC Report No. 1443. Research at Simon Fraser University was supported by the Research Corporation and the $\mathrm{Na}-$ tional Research Council of Canada.

†Present address. Physics Department, University of Mlinois, Urbana, ח1. 61801.

$\S$ Present address: Physics Department, Stanford University, Stanford, Calif. 94305. NSF Senior Postdoctoral Fellow on sabbatic leave from Cornell University 1970-71.

${ }^{1}$ R. D. Kirby, A. E. Hughes, and A. J. Sievers, Phys. Rev. B 2,481 (1970).

${ }^{2}$ R. D. Kirby, I. G. Nolt, R. W. Alexander, Jr., and A. J. Sievers, Phys. Rev. 168, 1057 (1968).

${ }^{3}$ I. G. Nolt and A. J. Sievers, Phys. Rev. 174, 1004 (1968); R. W. Alexander, Jr., A. E. Hughes, and A. J. Sievers, Phys. Rev. B 1, 1563 (1970).

${ }^{4}$ B. P. Clayman and A. J. Sievers, Phys. Rev. Letters 21, 1453 (1968).

${ }^{5}$ R. D. Kirby and A. J. Sievers, Solid State Commun. 6, 613 (1968).

${ }^{6}$ I. G. Nolt, R. D. Kirby, C. D. Lytle, and A. J. Sievers, Appl. Opt. $\underline{8}, 309$ (1969).

${ }^{7}$ B. P. Clayman, Ph. D. thesis, Cornell University, MSC Report No. 1026, 1969 (unpublished).

${ }^{8}$ H. D. Drew and A. J. Sievers, Appl. Opt. $\underline{8}, 2067$ (1969).

${ }^{9}$ G. Feher, I. Shephard, and H. B. Shore, Phys. Rev. Letters $\underline{16}$, 500 (1966). yielding $g=4.80 \times 10^{-3} \AA^{-6}$. Next, using the oscillator strength $^{11}$ of the mode $f=(6 \pm 2) \times 10^{-4}, \omega_{R}=120$ $\mathrm{cm}^{-1},{ }^{27}$ and $\mu=19.5 \mathrm{amu}$, we find

$$
\alpha_{\text {inic }}^{\prime}=0.6 \AA^{3} \text {. }
$$

The electronic polarizabilities of $\mathrm{Na}^{+}, \mathrm{I}^{-}$, and $\mathrm{Cl}^{-}$ yield

$$
\alpha_{\text {elec }}^{\prime}=6.21 \AA^{3},
$$

according to assumption (iii). The total polarizability for the mode is then $\alpha^{\prime}=6.81 \AA^{3}$. This value is used in Eq. (6) to get $E=2.4 E_{0}$.

${ }^{10}$ W. E. Bron and R. W. Dryfus, Phys. Rev. $\underline{163}, 304$ (1967).

${ }^{11}$ B. P. Clayman, I. G. Nolt, and A. J. Sievers, Solid State Commun. 7 , 7 (1969).

${ }^{12}$ W. Gebhardt, Phys. Rev. 159, 726 (1967).

${ }^{13}$ A. A. Kaplyanskii, Opt. i Spektroskopiya 16, 602 (1964) [Opt. Spectry. 16, 329 (1964)]; 16, 1031 (1964) [16, 557 (1964)].

${ }^{14}$ D. B. Fitchen, Physics of Color Centers, edited by W. B. Fowler (Academic, New York, 1968), Chap. 5.

${ }^{15}$ R. J. Elliott, W. Hayes, G. D. Jones, H. F. MacDonald, and C. T. Sennett, Proc. Roy. Soc. (London) A289, 1 (1965).

${ }^{16}$ W. Hayes and H. F. MacDonald, Proc. Roy. Soc. (London) A297, 503 (1967).

${ }^{17}$ A. J. Sievers, Localized Excitations in Solids, edited by R. F. Wallis (Plenum, New York, 1968), p. 27.

${ }^{18} \mathrm{~J}$. Tessman, A. Kahn, and L. W. Shockley, Phys. Rev. 92, 890 (1953).

${ }^{19}$ Lars Onsager, J. Am. Chem. Soc. 58, 1486 (1936).

${ }^{20}$ G. D. Mahan, Phys. Rev. 153, 983 (1967).

${ }^{21}$ R. J. Quigley and T. P. Das, Phys. Rev. 164, 1185 (1967); 177, 1340 (1969).

${ }^{22}$ W. D. Wilson, R. D. Hatcher, R. Smoluchowski, and G. J. Dienes, Phys. Rev. 184, 844 (1969).

${ }^{23} \mathrm{M}$. Gomez, Ph. D. thesis, Cornell University, MSC Report No. 910, 1968 (unpublished).

${ }^{24}$ I. Svare, Solid State Commun. 7, 1051 (1969).

${ }^{25}$ L. D. Landau and E. M. Lifshitz, Quantum Mechanics (Addison-Wesley, Reading, Mass., 1958).

${ }^{26}$ S. Roberts, Phys. Rev. 76, 1215 (1949).

${ }^{27}$ A. B. D. Woods, B. N. Brockhouse, R. A. Cowley, and W. Cochran, Phys. Rev. 131, 1025 (1967). 